\title{
RAWLS, ENTRE KANT Y HEGEL ${ }^{1}$
}

\author{
Carlos Peña \\ Universidad Diego Portales \\ Universidad de Chile
}

\begin{abstract}
Resumen / ABSTRACT
Ha llegado a ser un lugar común aseverar la influencia de Kant en la obra de Rawls; sin embargo, el constructivismo político (tal como se lo expuso en Political Liberalism, 1993) significó un rechazo del universalismo que es imposible explicar en términos kantianos. Lo que sigue es un intento de evaluar la tesis del consenso superpuesto a la luz de la concepción general de la filosofía política y la razón práctica en Hegel.
\end{abstract}

Palabras Clave: consenso superpuesto, liberalismo político, Rawls, Hegel, Kant, crítica descriptiva.

\section{RAWLS:BETWEEN KANT AND HEGEL}

It is a commonplace to affirm the kantian influence in the Rawls's work; however, the political constructivism (expose in Political Liberalism, 1993) meant an reject of universalism, that turn it is impossible to explain in kantian terms. What follows is an attempt of evaluating the overlapping consensus thesis in the light the general conception of political philosophy and practical reason in Hegel.

KEYWORDS: overlapping consensus, political liberalism, Rawls, Hegel, Kant, descriptive critique.

D1 En A Theory of Justice (1971), Rawls argumentó que mediante la razón era posible obtener un conjunto de principios de justicia cuya validez fuera independiente de toda contingencia. El camino para alcanzarlos fue un contrafáctico (que simulaba un pacto social en condiciones de incertidumbre) cuyos resultados puestos

Este artículo resume los resultados del Proyecto Fondecyt, 1020918, "Posibilidad y límites de la neutralidad política y filosófica en la obra de J. Rawls". Los resultados de esa investigación fueron antes expuestos en los siguientes lugares. Artículos: Peña, C. Rawls: equilibrio reflexivo, constructivismo y razón pública. El problema de la realidad y la justificación en filosofía política, en VVAA John Rawls. Estudios en su memoria, Revista de Ciencias Sociales, Universidad de Valparaíso, 2002, n 47, pp. 333-438. Libros: Peña, C.Rawls: el problema de la realidad y la justificación en filosofía política, México: Fontamara, 2008; Peña, C. Estudios sobre Rawls, Madrid: Coloquio Jurídico Europeo (con comentarios de H. Seleme y F. Vallespín), 2011. 
en relación con nuestros juicios ponderados permitían alcanzar un equilibrio reflexivo. En Kantian Constructivism in Moral Theory (1980) defendió las raíces kantianas de ese planteamiento presentándolo como una forma de constructivismo kantiano: una tesis antirrealista (Dummett 1990) de nuestros sentimientos morales. Así, él pretendía haber mostrado la fuerza moral del kantismo dentro de una teoría empírica (Rawls 1971: 251 y ss).

El constructivismo, como se sabe, es una tesis antirrealista para la cual el significado de un enunciado se reduce a las condiciones bajo las cuales lo consideramos suficientemente justificado (Dummett 1990: 244; cfr. O’Neill 2000). Para el constructivismo, la objetividad no depende de ninguna tesis asociada al realismo natural. En consonancia con esa tesis, la posición original se justificaría no porque describa o permita descubrir principios preexistentes, sino porque es la que mejor realiza la concepción que los hombres y las mujeres de una sociedad democrática tienen acerca de sí mismos como sujetos libres e iguales (Rawls 1999a: 223-252). Rawls desmintió, entonces, cualquiera interpretación epistemológica de la posición original, para sostener, en cambio, que se trataba de un procedimiento -en sus palabras, un device of representation- que realizaba la imagen moral que los sujetos de una sociedad democrática tienen acerca de sí mismos. Defendió, entonces, su propia concepción como un constructivismo kantiano; aunque no en rigor, sino por analogía (Ibíd.).

Ahora bien, esa filiación constructivista que Rawls reivindicó lo comprometió en un complejo debate filosófico que lo acercó al realismo interno de un autor como Putnam (vid, especialmente, Putnam 1994, 1992). Así se sigue de la caracterización que efectúa del constructivismo.

La concepción de Rawls del constructivismo kantiano deriva, en alguna medida, de la concepción de autonomía que sugieren las críticas de Sidgwick a Kant. Sidgwick sostuvo que si el concepto de autonomía se interpretaba neutralmente, entonces no sería posible distinguir la autonomía de un truhán de la de un santo (Sidgwick 1888: 411). De ahí Rawls derivó una concepción de autonomía de índole expresiva: obrar autónomamente equivale a actuar en base a principios que expresan nuestra condición de seres libres y racionales (Rawls 1971: 255).

El "constructivismo kantiano" afirma entonces que una teoría de la justicia o de la moralidad política es dependiente de la concepción de la persona (como agentes morales libres e iguales) (Rawls 1999a). Si la teoría moral fuera independiente de la concepción de la persona (y supusiera que hay un orden de objetos tal que determina lo que es justo con independencia de esa concepción), entonces, a juicio de Rawls, ella sería heterónoma. Esta concepción le permite a Rawls alejarse del intuicionismo que es, según este autor, una forma de realismo moral. Mientras el intuicionismo exige un sujeto cognoscente (que, mediante la intuición alcance los principios no derivados); el constructivismo kantiano exige una definición densa de persona como agentes morales libres e iguales (un concepto que, de otra parte, piensa Rawls, subyace en la cultura política democrática). Una justicia puramente procesal -como la que defiende Rawls bajo la forma de un constructivismo kantiano- rechaza la idea (que, por ejemplo, endosaría el utilitarismo bajo la forma de economía del bienestar) que: a. existen principios o 
entidades morales independientes; $b$. existe un procedimiento que asegura o hace más probable que, dado un caso, se logre una decisión derivada de esos principios. La justicia puramente procesal afirma, en cambio, que, dado un caso (v.gr., el diseño de las instituciones sociales básicas), no hay una solución justa independiente del procedimiento acordado para resolverlo y éste último, por su parte, es dependiente de un sistema de conceptos. En el mobiliario del universo no hay entidades - principios o figuras de otra índole- que equivalgan a, o de las que se deriven, principios de justicia o de moralidad política. Esos principios derivan de un procedimiento que, por su parte, es dependiente de la concepción que los agentes tienen acerca de sí mismos (lo que, como se ve, lleva a término el planteamiento de Sidgwick (1888). Rawls defiende la idea de que los principios de justicia son dependientes de una cierta concepción de la persona. Este punto de vista le permite, a la vez, rechazar el realismo moral del intuicionismo y pretender que sus propias afirmaciones son, sin embargo, objetivas (bajo el supuesto de que toda pretensión de verdad lo es al amparo de un cierto sistema de conceptos).

Esta caracterización de los principios formulados en Teoría de la Justicia posee una muy cercana relación con el realismo interno que, alguna vez, proclamó Putnam (Cfr. Putnam 1992). Putnam, como es sabido, defendió una forma de realismo que se distancia, por igual, del universalismo que se reprocha al liberalismo kantiano y el relativismo radical de un autor como Rorty (1999; 1991; 1989). En oposición al realismo metafísico (una versión de este tipo de realismo es el intuicionismo según lo caracteriza Rawls) Putnam sostiene que (a). los objetos y propiedades constitutivas son dependientes de un sistema de conceptos, y sostiene, además, esta vez en oposición al relativismo a la Rorty, que (b). hay muchos sistemas de conceptos verdaderos que describen correctamente el mundo (donde "verdadero" designa una aceptabilidad idealizada de nuestras proposiciones y no una correspondencia).

La tesis del constructivismo kantiano es compatible con ambas proposiciones. Así entonces, Rawls -en consonancia con los debates filosóficos generales- podría haber eludido los reproches al kantismo abstracto de su obra (Sandel 1998) por la vía de reivindicar una tesis de realismo interno. El realismo interno le permitiría salvar la objetividad de los juicios sin endosar la metafísica subyacente al intuicionismo (puesto que los juicios se confirman al interior de un sistema de conceptos) y eludir, al mismo tiempo, las acusaciones de universalismo abstracto (puesto que puede haber varios sistemas de conceptos). Una estrategia como esa le habría permitido, al mismo tiempo, elaborar una teoría de un alcance similar a la propuesta por Habermas pero con fundamentos más escuetos (Habermas 1995).

Sin embargo, no fue esa la estrategia seguida por Rawls en Political Liberalism (1993). En vez de asociar el constructivismo kantiano a alguna forma de realismo interno, lo sustituyó por lo que llamará constructivismo político.

Ahora la teoría se pretende neutra frente a las disputas doctrinales de la metafísica y sus consecuencias normativas reclaman validez solo para los ciudadanos de una democracia constitucional bajo condiciones modernas. Este punto de vista había sido anticipado en Justice as Fairness: Political not Metaphysical (1999d). La metafísica le parece allí a Rawls digna de rechazo no porque ella busque la unidad detrás de la 
diferencia, equipare el ser al pensamiento o confiera un carácter salvífico al pensamiento teórico (que son los motivos del alejamiento de Habermas 1990: 39) sino, simplemente, porque ella no asegura una orientación normativa común. La metafísica aparece ante todo a Rawls como una dispersión fragmentada de puntos de vista, especialmente doctrinales, que impiden el acuerdo y la fundamentación compartida de normas. Así concebida -como una disputa doctrinal en torno a los elementos últimos de la realidadla disputa metafísica se le antoja a Rawls una versión secular o laica de las guerras de religión a las que puso término el principio de tolerancia (Rawls 1993: xxvi).

Political Liberalism (Rawls 1993; Weinstock 1994: 165) no está entonces motivado por razones estrictamente conceptuales (si ese fuera el caso, y como se acaba de insinuar, él debió acercarse al realismo interno) sino por la estabilidad del sistema político al que la filosofía política se refiere (Rawls 1993: 38). En las condiciones modernas, sugiere Rawls, ninguna de las concepciones en juego puede garantizar-como no fuera al precio de la violencia o la intolerancia- la estabilidad del orden social. En esas condiciones, continúa, es imprescindible erigir a la tolerancia como el principio básico, poner en paréntesis las pretensiones de verdad y secularizar el entendimiento de la política (Thiebaut 1996). El resultado de ese esfuerzo sería la postulación de un consenso superpuesto.

Una interpretación como esa -la interpretación más o menos canónica en la literatura- priva, sin embargo, de consistencia al tránsito entre el constructivismo kantiano y el constructivismo político y deja pendiente (como ha sugerido buena parte de la literatura) la justificación de los principios de justicia. En efecto, mostrar la aceptación de los principios es una questio factis que no resuelve la questio juris: por qué esos principios deben ser aceptados.

La interpretación a la que se acaba de aludir deja expuesto a Rawls a lo que se ha llamado la crítica descriptiva. Esta crítica posee varias versiones; pero la más conocida es la que formuló Habermas (1995) en sus comentarios a Political Liberalism.

Habermas sostiene que la idea de consenso superpuesto separa el contenido en el que todos los ciudadanos pueden coincidir, de las razones por las cuales ese contenido les parece válido (1995: 122). Y así, el concepto de lo razonable-restringido, como se ve, solo al primer aspecto- queda tan deflacionado que es puramente descriptivo.

Así entonces, Rawls, para eludir la crítica descriptiva (versiones de ella hay también en Cohen 2003 y Raz 1990), debe hacer explícita alguna estrategia de fundamentación de los principios.

A la luz de la literatura (Stern 1993) hay al menos cuatro estrategias de justificación práctica. El fundacionalismo (la idea de que nuestras creencias actuales derivan de creencias básicas sin garantía inferencial como, por ejemplo, lo sugiere el intuicionismo que Rawls rechaza); la estrategia trascendental (el argumento del tipo modus ponens que emplean Kant o Habermas); el holismo teórico (como el de Quine); el holismo práctico (como el que se encuentra en Wittgenstein o Hegel) ¿A cuál de esas estrategias equivale la tesis del consenso traslapado? 
Si se hace pie en la interpretación canónica -conforme a la cual la tesis del consenso superpuesto intenta resolver el problema de la estabilidad-se arriba a la conclusión de que Rawls elude el problema de la justificación. A Theory of Justice estaría así por debajo de sus propias pretensiones: sería un caso de sociología puramente descriptiva. Una conclusión como esa es inaceptable para una teoría que, como la de Rawls, elabora un abundante contenido normativo.

Para resolver entonces el problema de la justificación -dar respuesta a la pregunta de por qué vale el contenido normativo que la teoría formula-sería necesario acreditar que la tesis del consenso superpuesto expuesto en Political Liberalism funda los principios de justicia sin incurrir en un non sequitur, es decir, sin derivar desde el plano de los hechos (desde la mera constatación de las convicciones que convergen en la sociedad democrática) una solución al problema de iure (el problema relativo a los principios que deben fundar la convivencia).

Para eludir esa falacia -y supuesto que se haya rechazado la estrategia fundacionalista- es imprescindible una estrategia holista: mostrar que una cuestión de facto (la convergencia que de hecho se produce en las sociedades modernas) resuelve el problema de iure (el problema de qué principios deben orientar la convivencia). En otras palabras, se hace necesario probar que una forma de vida (la sociedad moderna) tiene un contenido normativo ya realizado que solo se trata de explicitar. Una perspectiva como esa exige rechazar cualquier estrategia trascendental (como la del kantismo que suele adscribirse al argumento de Rawls) y, en cambio, "historizar" aquello que puede contar como apelación a razones (que es la estrategia seguida por Hegel; vid. Pippin 2010, 2008). En otras palabras, la postulación del consenso superpuesto incurriría en un non sequitur (otra versión de uno de los varios reproches que Hegel dirige a Kant; Hegel 2006: 704 y ss; cfr. Taylor 2010: 325; Hyppolite 1998: 430) a menos que se funde en una concepción de la racionalidad práctica conforme a la cual no hay distancia entre lo que debe ser y lo que es, algo que solo puede alcanzarse con un argumento a la Hegel (Cfr. Pippin 2008). Pero si eso es así, entonces la tarea de la filosofía política no es la de legislar con afanes de universalidad, sino simplemente la de reconocer lo que ya estaba allí (Hegel 2000).

Un examen de los textos de Rawls, incluidas sus Lectures de filosofía general, y una discusión con la literatura secundaria, permite dar plausibilidad a la precedente hipótesis. En efecto, la tesis del consenso superpuesto puede ser presentada como una descripción del potencial normativo que subyace a la reflexiva pluralidad moderna y a la filosofía política que la proclama como un esfuerzo por reconciliar a los miembros de las sociedades modernas con sus propias instituciones.

Existe, desde luego, abundante evidencia textual de que Rawls se dejó influir por Hegel tal cual él era presentado en la academia anglosajona por Hardimon (1994) y por Avineri (1972). El rastro más evidente de esa influencia es la forma en que Rawls concibe su propio trabajo filosófico: como una tarea de reconciliación, alejándose así de la concepción que de la filosofía, como custodia y aseguradora de los límites y posibilidades de la razón, tenía Kant; pero también se encuentra el hecho de que para Rawls, al igual que Hegel, la estructura básica de la sociedad es el principal objeto de 
la filosofía política y el obvio paralelo entre el concepto de unión social rawlsiana y el de eticidad hegeliana (Schwarzenbach 1991; Hardimon 1994: 10; 1992: 166; y el más reciente, Bercuson 2014: 30 y ss ).

Pero no alcanza ahí la influencia de Hegel en Rawls. Todavía puede sostenerse que la estructura del razonamiento práctico en Rawls posee una impronta hegeliana. Hegel ayudaría, de esta forma, a superar la crítica descriptiva.

El concepto central en la estructura del razonamiento práctico, tal como lo concibe Rawls, es el de "equilibrio reflexivo". El concepto fue acuñado por Nelson Goodman, a propósito de las inferencias inductivas. Hume, sugirió Goodman, habría resuelto bien el problema de las inferencias inductivas: nuestras inferencias las corregimos a la luz de nuestras prácticas inferenciales y estas por su parte las estimamos correctas cuando nos conducen a inferencias particulares que no estamos dispuestos a desechar. Se trata, se ha observado, de una concepción de innegable tinte aristotélico, puesto que no tiene por objeto socavar las opiniones establecidas (Hampshire 1984 ).

A la luz de lo anterior, la posición original de Rawls no es un mecanismo neutro, de pura racionalidad neoclásica, sino, como el propio Rawls va a subrayar, se trata de un mecanismo de representación, un artificio para estilizar las concepciones de hecho existentes en la sociedad, y poder luego contrastarlas mediante el equilibrio reflexivo. El razonamiento comienza seleccionando un conjunto de concepciones puramente políticas que subyacen en la cultura pública (a esto Rawls lo llama justificación pro tanto). Cuando se las estiliza equivalen a la tradición liberal y la democrática, una acentuando la autonomía individual y la otra, la colectiva. Esta parte del razonamiento le corresponde a la filosofía moral, cuya tarea sería descriptiva de lo que subyace en la historia constitucional, sin prejuzgar asuntos ni ontológicos, ni epistemológicos. El resultado es equilibrado por cada una de las cosmovisiones en juego dentro de la pluralidad moderna, cada una de las cuales busca razones esta vez internas a ellas (ya no puramente políticas) para converger. El resultado es el consenso superpuesto que permite un ámbito de razones públicas que todos pueden compartir y que mantienen las razones internas sin abandonarlas, aunque en la sombra.

Schwarzenbach ha insinuado, por eso, que el "equilibrio reflexivo" al que echa mano Rawls es, a fin de cuentas, un mecanismo de reconciliación en el sentido hegeliano de la expresión. Para el constructivismo de Rawls, los principios de la moralidad política subyacen a la cultura pública (un concepto, sugiere Schwarzenbach, equivalente al de "espíritu objetivo") de suerte que el equilibrio reflexivo sería una tarea inferencial que equivale a una suerte de autoconocimiento de lo que ya está ahí. El equilibrio reflexivo poseería así un indudable tinte hegeliano, sobre todo porque, sugiere Schwarzenberg, supone superar la negatividad de nuestros juicios o de las concepciones subyacentes en una serie sucesiva de superaciones o metalenguajes que recuerdan a la Lógica de Hegel (que, por supuesto, posee un tinte innegablemente aristotélico, Marcuse 1972: 124 ; Cfr. Stuart Hampshire 1984).

El problema más obvio al que esa estrategia de justificación debe hacer frente es la acusación de suprimir toda dimensión crítica en el análisis. Si las concepciones que llevan a cabo la justificación pro tanto se estilizan a partir de las que subyacen en 
la cultura pública y si, luego, culminan en el consenso por superposición ¿cuál es el patrón normativo que permitiría distinguir la questio juris de la questio factis?

Ese problema es, por supuesto, similar al que se plantea con el famoso Dopplesatz hegeliano, según el cual lo actual es racional y lo racional actual².

Rawls se pronunció acerca de la probable supresión del plano crítico en el análisis hegeliano que subyace a la filosofía como reconciliación. En su opinión, el mero desarrollo histórico no justifica por si solo el patrón normativo que le subyace. Ha de haber, sugiere, algún bien final en el sentido aristotélico cuya búsqueda sirva como razón. Ese tipo de bien es un logro colectivo y por eso al mirar hacia atrás el Geist puede constatarlo en el curso de la historia (Rawls 2000: 371). Esta sugerencia de Rawls, que es también una respuesta a la crítica descriptiva que su tesis ha recibido, es coherente con una concepción, a la Hegel, del compatibilismo entre naturaleza e historia.

Para Hegel, la distinción entre naturaleza y cultura no es ontológica sino en sí misma histórica y normativa, depende de una norma social que ha sido históricamente formulada (Pippin 2008: 65). En otras palabras, la racionalidad práctica es interna al Geist lo que significa que los dualismos no existen y cualquier forma de realismo moral carece de sentido (Rawls; Pippin 2008: 55). Kant y Hegel comparten entonces un mismo proyecto: el valor y la dignidad humana derivan del hecho de no estar sometidos a otra ley o principio de acción que el que los seres humanos se dan a sí mismos (Pippin 2008: 65; 1999: 18). Ambos piensan entonces que los roles e instituciones que existen de hecho no proveen razones o principios para actuar a menos que hayan pasado el test reflexivo de la subjetividad (que es lo que Hegel considera el principio básico de la modernidad, como lo expresa en el parágrafo 124 de su Filosofía del Derecho). Con todo, para que

2 En la literatura hegeliana el sentido de ese Doppelsatz se ha discutido entre quienes le asignan un sentido conservador (coincidiendo con el reproche de que es objeto la crítica descriptiva) o quienes, en cambio, le asignan un sentido crítico (permitiendo entonces escapar de ese reproche). Un ejemplo de la interpretación conservadora del Doppelsatz es el de Popper (1966: 41). Este autor interpreta el doble dictum como si en él se dijera "aquello que es, por el hecho de serlo es bueno". No habría en Hegel, interpreta Popper, un estándar más alto que el último desarrollo de la razón o de la idea y así entonces toda normatividad crítica desaparece. Por la inversa, quienes se oponen a esa interpretación, sugieren que ella malentiende el término empleado por Hegel. Actualidad (Wirklichkeit) alude no solo a lo que existe sino a lo que es conforme a su esencia. Para él entonces no basta existir para ser bueno, puesto que hay cosas que meramente existen que no lo son en modo alguno. Así entonces el dictum hegeliano no sería conservador, puesto que llamaría la atención acerca del vacío que media entre lo que meramente existe y la esencia que debe ser realizada (Hardimon 1994: 59). Como explica Stern (2006: 238), ambas interpretaciones parten de una base común: la idea de que el dictum posee un contenido normativo, solo que en un caso (la interpretación conservadora) ese contenido estaría ya realizado, en tanto que para la otra (la interpretación crítica de Hegel) estaría por realizarse. Enfrente de ambas interpretaciones, Stern sugiere que el uso del término racional en el dictum hegeliano es meramente metodológico y alude a la racionalidad de la tarea filosófica. Lo actual es racional porque, en opinión de Hegel, podría ser inteligido. 
una acción cuente como mía no basta el endoso puramente individual, como explica Hegel al criticar a Kant (Pippin 2008: 69; 1991), sino que ella tiene que ser inteligible al interior de un complejo de prácticas e instituciones, hacer sentido con la totalidad de una cultura pública en los términos de Rawls (Pippin 2010; 2008: 265; 1991).

El consenso superpuesto -al igual que el famoso Dopplesatz hegeliano- no sería entonces un mero factum del que los principios no podrían derivarse: el consenso superpuesto de la sociedad moderna y democrática revelaría un logro colectivo de la agencia moral con el que la filosofía política - esa filosofía que según Rawls, deja a la filosofía tal como está- logra reconciliarnos.

\section{Referencias bibliográficas}

Avineri, S. (1972), Hegel's Theory of the Modern State. Cambridge: Cambridge University Press.

Bercuson, J. (2014), John Rawls and the History of Political Thought. The Rousseauvian and Hegelian Heritage of Justice as Fairness. Routledge.

Cohen, G. (2003), "Facts and Principles Author(s)", Philosophy and Public Affairs, Vol. 31, N 3, pp. 211-245.

37 , Issue 1.

(2008), "Truth and Public Reason", Philosophy and Public Affairs, Vol.

Dummett, M. (1990), La verdad y otros enigmas. México: FCE.

(1990), "The source of the concept of truth", Meaning and Method.

Essays in Honor of Hilary Putnam. Cambridge: Cambridge University Press.

Goodman, N. (1978), Ways of Worldmaking. Paperback.

(2004), Hecho, ficción y pronóstico. Madrid: Editorial Síntesis S.A.

Habermas J. (1995), "Reconciliation through the public use of reason: remarks on John Rawls's political liberalism". The Journal of Philosophy. March 1995, v. 92 n³ p. 109 (23).

(1990), Pensamiento postmetafísico. México: Taurus, Humanidades.

Hampshire, S. (1984), Dos teorías de la moralidad. México: FCE.

(1972), "A Theory of Justice, by J. Rawls", A Special Supplement: A New Philosophy of the Just Society. New York Books of Review, 24 de febrero 1972.

Hardimon, M. (1992), “The Project of Reconciliation: Hegel's Social Philosophy”, Philosophy and Public Affairs 21, 2. 165-195.

(1994), Hegel's Social Philosophy. The Project of Reconciliation.U.K.: Cambridge University Press.

Hedrick, Todd (2010), Rawls and Habermas. Reason, Pluralism and the Claims of Political Philosophy. California: Stanford University Press. 
Hegel, G.W.F. (2000), Rasgos Fundamentales de la Filosofía del Derecho o Compendio de Derecho Natural y Ciencia del Estado. Madrid: Biblioteca Nueva.

(2006), Fenomenología del Espíritu (Traducción de Manuel Jiménez Redondo). Valencia: Pre Textos.

(1981), Lecciones sobre Filosofía del Derecho. B. Aires: Universidad de Morón. Porrúa.

(2004), Enciclopedia de las ciencias filosóficas. México: Editorial

Hyppolite, J. (1998), Génesis y estructura de la Fenomenología del Espíritu de Hegel. Barcelona: Península.

Marcuse, H. (1972), Razón y Revolución. Hegel y el surgimiento de la teoría social. Madrid: Alianza.

O'Neill, O (1997), "Political liberalism and public reason: a critical notice of John Rawls, 'Political Liberalism", The Philosophical Review. July 1997, v. 106 n³, p. 411 (18).

Press.

(2000), Constructions of Reasons. Cambridge: Cambridge University

(1989), "The public use of reason", Constructions of Reason. Explorat ions of Kant's Practical Philosophy. Cambridge: Cambridge University Press, pp. 28-51.

Peperzak (1987), Philosophy and Politics: A Commentary on the Preface to Hegel's "Philosophy of Right" (International Archives of the History of Ideas). Springer.

Pinkard (2008), Hegel. Una biografía. Madrid: Acento.

Pippin, R. (1991), Idealism and Agency in Kant and Hegel, The Journal of Philosophy, Vol. 88, $\mathrm{N}^{\circ}$ 10, Eighty Eight Annual Meeting American Philosophical Association, Eastern Division, pp. 532-541.

(1989), Hegel's Idealism. The Satisfactions of Self-Consciousness. U.K.: Cambridge University Press

(2008), Hegel's Practical Philosophy: Rational Agency as a Ethical Life. Paperback.

(2010), "La justificación por desarrollo: la idea de una lógica de la experiencia en la Phänomenologie des Geistes de Hegel, en Lemm y Ormeño (edit.), Hegel, pensador de la actualidad. Santiago: UDP.

Pippin, R. and Höffe, Ottfried (editors.) (2003), Hegel on Ethics and Politics. U.K.: Cambridge University Press

Popper, Karl (1966), The Open Society and Its Enemies, Volume II: The High Tide of Prophecy: Hegel, Marx and the Aftermath, $5^{\text {th }}$. Ed. London: Routledge.

Putnam, H. ( 2002), The collapse of the fact/value dichotomy and other essays. Harvard University Press. 
(1992), A defense of internal realism, Realism with a human face. Cambridge: Harvard.

(1983), "Reference and Truth, in Realism and Reason", Philosophical Papers, Vol. 3, Cambridge.

(1995), Words and life. Harvard.

(1994), Las mil caras de la realidad. Buenos Aires: Paidós.

(1994), Las mil caras del realismo. Buenos Aires: Paidós, 161 pp., The

Paul Carus Lectures. Washington, 1985, Open Court, La Salle III, 1987.

( 1988), Razón, verdad e historia. Madrid: Tecnos.

(1994), "Senses, nonsense and the senses: an enquiry into the powers of the human mind", The Journal of Philosophy Vol. 91, № 9, pp. 445-517.

(1975), "The meaning of meaning", Philosophical papers, Volume 2. Mind, language and reality, pp. 215-271. Cambridge University Press.

Quine, W. (1969), "Epistemology naturalized", Ontological Relativity and other essays. Columbia.

( 1997), La búsqueda de la verdad. Barcelona: Crítica.

(1991), "Dos dogmas del empirismo", en Valdés (ed.), La búsqueda del significado. Tecnos. cit.

Rawls, J. (1971), A Theory of Justice. Harvard University Press.

( 1993), Political Liberalism. Columbia.

(1995), Teoría de la justicia. México: F.C.E.

(1995a), Reply to Habermas.

(Freeman, ed.) (1999), Collected Papers. Harvard University Press.

(1999), The law of peoples. Harvard University Press.

(2000), Lectures on the History of Moral Philosophy. Harvard: University

Press.

(2001), Justice as fairness. A restatement. Harvard University Press.

( 2007), Lectures on the History of Political Philosophy. Harvard University Press.

(1999a), "Kantian Constructivism in Moral Theory", Collected Papers, editados por S. Freeman. Cambridge: Harvard University Press.

(1999b), "The Independence of Moral Theory", Collected Papers, editados por S. Freeman. Cambridge: Harvard University Press.

(1999c), “A Kantian Conception of Equality", Collected Papers, editados

por S. Freeman. Cambridge: Harvard University Press.

(1999d), "Justice as Fairness: Political not Metaphysical”, Collected Papers, editados por S. Freeman. Cambridge: Harvard University Press.

Paidós.

(2007), Lecciones sobre la historia de la filosofia moral. Buenos Aires: 
( 2000), Lectures on History of Moral Philosophy, en Barbara Herman, ed. Harvard University Press.

(1996), El Liberalismo Politico. Barcelona: Crítica.

Raz, J., (1990), "Facing Diversity: The Case of Epistemic Abstinence", Philosophy and Public Affairs, Vol. 19, Issue 1 (Winter, 1990), pp. 3-46.

Rorty (1999), “A World without correspondence to reality", Philosophy and Social Hope. Penguin Books.

(1991), "Objectivity, Relativism and Truth", Philosophical Papers. Cambridge University Press.

(1989), Contingency, Irony and Solidarity. Cambridge University Press. (1979), La filosofía y el espejo de la naturaleza. Cátedra, 1995.

(1996), "Pragmatismo, Davidson y la Verdad", Objetividad, relativismo $y$ verdad. Paidós.

(2000), "Universality and Truth", Brandom, R. (ed.), Rorty and his Critics. Oxford: Blackwell.

( 1996), Objetividad, relativismo y verdad. Barcelona: Paidós.

(2007), What's the Use of Truth? New York: Columbia University Press.

Sandel, M. (1998), Liberalism and the Limits of Justice. Cambridge University Press.

Schwarzenbach, S. (1991), "Rawls, Hegel, and Communitarianism", Political Theory, Vol. 19, No. 4 (Nov., 1991), pp. 539-571.

Sidgwick, H. (1888), “The Kantian Conception of Free Will”, Mind, vol. 13, n 51 (Jun).

Stern, D. (1993), "Foundationalism, Holism or Hegel", G. W. Hegel: Critical Assesments. London: Routledge.

(2006), "Hegel's Doppelsatz: A Neutral Reading", Journal of the History of Philosophy, Volume 44, Nr. 2, April 2006, pp. 235-266.

Taylor, Ch.(1995), "Cross-Purposes: The liberal-communitarian debate”, Philosophical arguments. Cambridge: Harvard, p. 182.

(2010), Hegel. Barcelona: Anthropos.

Thiebaut (1996), "El espacio político de las diferencias, Recensión de J. Rawls, El liberalismo político", Revista de Libros, Caja de Madrid, número O, (Diciembre).

Weinstock Daniel M. (1994), "The justification of political liberalism (Special Double Issue: John Rawls's ‘Political Liberalism')”. Pacific Philosophical Quarterly, Sept-Dec 1994 v75 n³ 3-4 p. 165 (21). 